\title{
Expression of Tumour-Associated MUC1 Is a Poor Prognostic Marker in Breast Cancer in Kumasi, Ghana
}

\author{
E. Atta Manu, ${ }^{1}$ K. Bedu-Addo $\mathbb{D}^{1},{ }^{1}$ N. A. Titiloye, ${ }^{2}$ C. Ameh-Mensah, ${ }^{1}$ F. Opoku, ${ }^{1}$ \\ and B. M. Duduyemi $\mathbb{D D}^{2}$ \\ ${ }^{1}$ Department of Physiology, Kwame Nkrumah University of Science and Technology, Kumasi, Ghana \\ ${ }^{2}$ Department of Pathology, Kwame Nkrumah University of Science and Technology, Kumasi, Ghana \\ Correspondence should be addressed to B. M. Duduyemi; tundeduduyemi@gmail.com
}

Received 22 January 2020; Revised 23 March 2020; Accepted 2 April 2020; Published 22 April 2020

Academic Editor: Guido Bocci

Copyright (C) 2020 E. Atta Manu et al. This is an open access article distributed under the Creative Commons Attribution License, which permits unrestricted use, distribution, and reproduction in any medium, provided the original work is properly cited.

Background. Immunohistochemical assessment of breast cancer and stratification into the basic molecular subtypes afford a much deeper insight into the biology of breast cancer, while presenting with opportunities to exploit personalized, targeted treatment. Traditionally, the oestrogen, progesterone, and epidermal growth factor receptors are assessed. MUC1, a transmembrane mucin, has been demonstrated a potential prognostic and metastatic marker in breast cancer. However, there have been a limited number of studies addressing the predictive and prognostic features of MUC1 in African breast cancer. This study aims at addressing the expression profiles of MUC1 and other biomarkers in Ghanaian breast cancer and determines its predictive and prognostic characteristics, in relation to other clinicopathological features. Methods. Haematoxylin and eosin (H\&E) slides of breast cancer cases were reviewed and 203 suitable cases were selected for tissue microarray (TMA) construction and immunohistochemistry. Anti-ER, PR, HER2, Ki-67, and MUC1 antibodies were used. Results from the immunostaining were analysed using SPSS version 23. Results. About $59 \%$ of cases expressed MUC1. Majority of cases in the study showed a lack of expression of all three traditional markers (29\% expressed ER, 10.9\% PR, and 20.7\% HER2). Ki-67 index were 62.1\% (low), 16.5\% (moderate), and 21.4\% (high). MUC1 expressions among the molecular classes were luminal A (60.7\%), luminal B (68.8\%), HER2 overexpression (87.5\%), and triple negative $(56.6 \%)$. There were significant associations between MUC1 and HER2 overexpression $(p=0.01)$ and triple negative $(p<0.01)$. Conclusion. The high proportion of breast cancer cases expressing MUC1, as well as its association with the two most aggressive molecular classes, indicate a substantial role in the biology of breast cancer in our cohort, and it is an indication of poor prognosis.

\section{Introduction}

Breast cancer, the most commonly diagnosed cancer type in women globally, has remained an important health challenge for decades. With an estimate of 2 million newly diagnosed cases and a corresponding 626,679 deaths in the year 2018, breast cancer has proven to be a major barrier to improvements in life expectancy worldwide [1].

Appreciable improvements have been made in the diagnosis, treatment, or management of breast cancer, especially in the developed countries [2-4]. The frequent occurrence of hormone-positive breast cancers among whites in these developed countries [5-7], and the administration of targeted therapies that antagonize the activity of oestrogen and/or progesterone such as tamoxifen after adjuvant chemotherapy, has led to great reductions in the breast cancer-specific mortality rates in these countries [8].

Additionally, the use of trastuzumab, pertuzumab, and other therapies for HER2+ breast cancer has contributed immensely towards an improvement in the overall survival of breast cancer patients [9-11]. Consequently, percentages of 5-year survival with breast cancer are documented to be over $80 \%$ in the United States and Europe $[4,12,13]$.

In Africa, however, alarming increase in the incidence of breast cancer [1], which is mostly of aggressive histological 
characteristics and frequent lymph node metastasis, presents a major health challenge to women. This challenge is compounded by issues associated with access to healthcare, diagnosis, treatment, and management of the disease, especially in low-resource settings $[2,14,15]$.

Central to the challenges faced in the treatment and management of breast cancer in Africa is the fact that African breast cancer exhibits distinct molecular characteristics from that presented by Caucasians [16]. Although variations in frequencies have been reported across the continent, African women are known to present with the highest proportions of receptor-negative or triple-negative breast cancers $[2,14,17]$. This indicates that a substantial proportion of African women diagnosed of breast cancer are unable to benefit from anti-ER nor anti-HER2 adjuvant therapies and, in the absence of alternative molecular targets, must resort to conventional chemotherapy. In line with the assertion that triple-negative breast cancers are a heterogenous group, there are variable responses to administered chemotherapy, and the outcome for a number of cases are still unfavourable $[18,19]$.

Consequently, survival among African women with breast cancer is much lower compared to that of Whites, with as low as $13.6 \%$ in Gambia [20]. In line with the lower rates particularly in sub-Saharan Africa, a recent study on survival outcomes of breast cancer in Ghana demonstrated that the overall 5-year survival was $47.9 \%$ [21]. This underscores the need for further biomarker research to identify predictive/prognostic markers which may be amenable for improved treatment.

MUC1, a single-pass, type 1 transmembrane glycoprotein that is expressed only on the apical surfaces of normal glandular and ductal epithelial cells [22, 23], has been shown to be a potential target for cancer therapy, ranking as the $2^{\text {nd }}$ cancer vaccine target among 75 other cancer antigens assessed by the National Cancer Institute [24]. This rank was based on the tumour-specific aberrant glycosylation pattern of MUC1 in various forms of adenocarcinoma. The hypoglycosylated MUC1 is overexpressed and is expressed on the whole cell surface, in contrast to the apical expression on normal cells [25]. Because of the aberrant glycosylation, T- and B-cell epitopes in the peptide backbone are now accessible, forming tumour-associated MUC1 antigens [23, 26]. Accordingly, a number of clinical and preclinical studies have delved into MUC1-mediated cancer immunotherapy and vaccination (reviewed elsewhere [27-29]).

Expectantly, studies have been designed to assess the predictive and prognostic importance of MUC1 in various forms of cancer [30], including invasive and metastatic breast cancer [31-33], with the aim of establishing a basis for improved diagnosis and the potential benefits MUC1 immunotherapy could afford. Although MUC1 is overexpressed in the majority of breast cancer cases studied $[13,32-36]$, there appears to be racial differences in the proportion of positives and associated prognosis. Despite this variation, studies of MUC1 in African breast cancer have been sparse, and currently, no single such study has been done here in Ghana.
This study is designed to assess the proportion of MUC1 positives and establish the predictive and prognostic significance of MUC1 in primary breast carcinoma presented by Ghanaian women in Kumasi.

\section{Materials and Methods}

2.1. Ethical Consideration and Study Samples. Following approval (REG NO:RD/CR18/203) from the Research and Development Unit, Komfo Anokye Teaching Hospital (KATH), ethical approval (Ref:CHREP/AP/417/18) was obtained from the Committee on Human Research Ethics and Publication, Kwame Nkrumah University of Science and Technology (KNUST), under the title "Molecular Profiling of Breast Cancer in Kumasi” prior to execution of the study protocol.

The study population comprised consecutive cases of female patients visiting the breast clinic at KATH from 2009 to 2017, with primary breast cancer tissues preserved as formalin-fixed paraffin embedded (FFPE) blocks. Patient demographics were abstracted from a database at the Department of Pathology, KATH, where the samples had been sent for histopathological appraisal. The information included age, sex, histological diagnosis, tumour grade, and lymphovascular invasion.

Haematoxylin and eosin- (H\&E-) stained slides were made from the FFPE blocks for joint review by two pathologists (NAT and BDM). Review was made according to guidelines outlined by the WHO [37]. Clinicopathological data were confirmed or amended where applicable. The most representative tumour areas were marked on the H\&E slides to aid in the construction of a tissue microarray (TMA).

2.2. Tissue Microarray (TMA) Construction. TMA was constructed with slight modifications to the procedure described previously [38]. Briefly, recipient paraffin blocks were constructed using a silicone mould supplied with a manual TMA machine (Micatu MicaArray Gen. 4). Following the delineation of a TMA map according to the case identification numbers, the FFPE tissue blocks (henceforth referred to as "donor blocks") were oriented and the representative tumour foci identified with the aid of the reviewed H\&E slides. Successively, two cylindrical cores (diameter: $1.0 \mathrm{~mm}$ each) of tissue were extracted from each donor block and inserted into predrilled holes in the TMA recipient block. After insertion of the tissue cores, the recipient block was heated gently under an incandescent lamp to allow the tissue cores to sink in further.

2.3. Immunohistochemistry (IHC). Immunohistochemical staining was performed according to standard procedures. Antibodies for cyclin D1, estrogen receptor (ER), HER2, Ki67, MUC1, and progesterone receptor (PR) were used. $3 \mu \mathrm{m}$ thick sections were made from each TMA block onto SuperFrosted Plus slides and taken through deparaffinization using xylene. The sections were subsequently rehydrated using a series of ethanol solutions of decreasing grades (absolute-95\%-70\%), diluted with tris buffered saline 
(TBS), and washed with distilled water. Antigen retrieval was performed by incubating the TMA sections in citrate buffer $(\mathrm{pH} 6)$ in a pressure cooker $(10 \mathrm{~min})$. Background staining and nonspecific antibody binding were prevented using hydrogen peroxide (3\%) in methanol for 10 minutes and casein solutions, respectively. The sections were incubated with the primary antibodies according to the manufacturers' specifications (shown in Table 1). Secondary antibody conjugated with peroxidase and anti-peroxidase (DAKO) was added. Sections were developed later with diaminobenzidine tetrahydrochloride (DAB). The sections were counterstained in haematoxylin, dehydrated in increasing grades of ethanol (70\%-95\%-absolute), and mounted using the DPX mountant.

2.4. Scoring of IHC. IHC-stained sections were scored according to percentages of cells positive for the various biomarkers.

2.5. Statistical Analysis. Records on patient demographics, clinicopathological characteristics, and results from IHC were analysed using the Statistical Package for Social Sciences (SPSS) version 23. Associations in expression of biomarkers and clinicopathological characteristics and demographics were explored using the chi-squared test. A $p$-value $\leq 0.05$ at the $95 \%$ confidence level was considered statistically significant.

\section{Results}

A total of 203 cases had preserved blocks with representative tumour foci for the study period, hence formed the study samples. Descriptive statistics of the cases' demographics and histological characteristics are detailed in Table 2. The mean age of the cases was 49.34 years, and invasive carcinoma NST was the predominant histological type (83.0\%). Majority of the cases were of histological grade III (55.7\%), while $50.8 \%$ were negative for lymphovascular invasion.

3.1. Immunohistochemical Characteristics. Majority of the cases were negative for expression of ER, PR, and HER2. The respective negative percentages are 71.0, 89.1, and 79.3. MUC 1 was rather overexpressed in majority of cases, with a percentage of 59.0. Table 3 details the immunohistochemistry results of the biomarkers, along with $\mathrm{Ki}-67$, for which $62.1 \%$ of cases had a low expression, and cyclin D, with 44.8 positive cases. Selected photomicrographs of positive cases for each biomarker and control cases for MUC 1 are displayed in Figures (1) and (2). Based on the expression of ER, PR, HER2, and Ki-67, the cases were categorized into the breast cancer molecular subtypes. The triple-negative molecular subtype formed the largest group, constituting 54.3\% of cases.

3.2. Statistical Analysis. The chi-squared test was computed for association between MUC1 and the molecular subtypes, other markers, and the clinicopathological characteristics.
Table 4 shows the percentages of cases of each parameter or marker which were positive for MUC1 expression, along with the chi-squared values and associated $p$ values. Significant associations were observed between MUC1 overexpression and cyclin D $(p<0.001)$, HER2 overexpression $(p=0.01)$, and triple negative $(p<0.05)$.

\section{Discussion}

This study sought to assess the predictive and prognostic significance of MUC1 in breast cancer presented by Ghanaian women in Kumasi. Various such studies have been conducted on breast cancer samples from the developed world, where it is overexpressed in a very high proportion of cases and is generally associated with good prognosis. Studies on MUC1 in African breast cancer samples are virtually nonexistent, as only a handful has been done. This is one of the first such studies in Ghana and hence provides novel information about the biology of breast cancer here, and by extension, West Africa.

The results indicate that the larger proportion of cases analysed overexpressed MUC1. This is in line with the longheld assertion that MUC1 is widely expressed in all forms of adenocarcinoma [23]. The proportion of MUC1 positives (59.0\%) observed in the current study is however lower, compared to most Western studies, by stressing differences between breast cancer in African and Caucasians. Proportions of MUC1-positive cases reported by Western studies range from $86.4 \%$ [35] to $100 \%$ [30]. It is however in consensus with the 58\% reported by Patel et al. [39] in a cohort of Indian cases. Elseed et al. [40] recorded 72.5\% MUC1-positives in a study on Sudanese cases, the only prior African study on the prognostic significance of MUC 1 accessible to us.

The association between MUC1 overexpression and age and histological diagnoses revealed no significant statistical values ( $p=0.810$ and 0.533 , respectively). Likewise, no significant associations were observed between MUC1 positivity and histological grade. It was however observed that MUC1-positive cases were predominantly of higher grades (Table 4). This contrasts the published literature on western cases, where MUC1 positivity is often associated with lower histological grades [32, 34-36]. Again, no significant association was observed between MUC1 overexpression and $\mathrm{Ki}-67$, yet MUC1-overexpressing cases were predominantly of high Ki-67 indexes, suggesting higher mitotic activities in these tumours.

It was observed that MUC1 overexpression was strongly associated with aberrant expression of cyclin $\mathrm{D}(p<0.001)$. Cyclin D is a cell-cycle protein involved in the progression through the G1 stage to the $S$ phase. Its overexpression in cancers has been described to be associated with enhanced proliferation rates, poor prognosis, and decreased survival [41]. Liu et al. [42] demonstrated expression of aberrant glycosylated MUC1 and activity induced sustained expression and stability of cyclin $\mathrm{D}$, thereby promoting proliferation. Our findings on the association with cyclin D overexpression supports a study by Van Der Vegt et al. [33], who found a similar significant association between 
TABle 1: Details of antibodies used.

\begin{tabular}{lcccccc}
\hline Antibody & Clone & Pretreat & Dilution & Control & Company & Address \\
\hline CYCLIN-D1 & SP4 & ER2/20 & $1: 40$ & $88-13792-7 A$ & THERMO SCIENTIFIC & Grandy Island, NY \\
ER & 1D5 & ER1/20 & $1: 50$ & Breast CA & BioCare Medical & Concord, CA \\
HER-2 & & ER1/20 & RTU & Breast CA & DAKO & Carpinteria, CA \\
Ki-67 & MIB-1 & ER1/20 & $1: 80$ & Tonsil & DAKO & Carpinteria, CA \\
MUC-1 & Ma695 & & $1: 160$ & Colon CA & Leica Microsystems & Buffallo Grove, IL \\
PR & PgR 636 & ER1/10 & $1: 400$ & Endo/Myome & DAKO & Carpinteria, CA \\
\hline
\end{tabular}

TABle 2: Descriptive statistics of TMA cases.

\begin{tabular}{|c|c|c|}
\hline Age distribution & Mean/years & Standard deviation/years \\
\hline & 49.34 & 13.739 \\
\hline \multirow{9}{*}{ Histological diagnoses } & Type & Frequency (percentage) \\
\hline & Invasive carcinoma NST & $166(83.0)$ \\
\hline & Ductal carcinoma in situ & $9(4.5)$ \\
\hline & Metaplastic carcinoma & $6(3.0)$ \\
\hline & Invasive lobular carcinoma & $5(2.5)$ \\
\hline & Mucinous carcinoma & $5(2.5)$ \\
\hline & Invasive papillary carcinoma & $2(1.0)$ \\
\hline & Medullary carcinoma & $2(1.0)$ \\
\hline & Others & $5(2.5)$ \\
\hline \multirow{3}{*}{ Histological grade } & Grade I & $14(9.4)$ \\
\hline & Grade II & $52(34.9)$ \\
\hline & Grade III & $83(55.7)$ \\
\hline \multirow{2}{*}{ Lymphovascular invasion } & Positive & $32(49.2)$ \\
\hline & Negative & $33(50.8)$ \\
\hline
\end{tabular}

TABle 3: Immunohistochemical characteristics of the cases.

\begin{tabular}{lcc}
\hline Biomarker & Frequency & Percent \\
\hline ER & & \\
$\quad$ Positive & 54 & 29.0 \\
Negative & 132 & 71.0 \\
\hline PR & 20 & \\
$\quad$ Positive & 163 & 10.9 \\
Negative & & 89.1 \\
\hline HER2 & 37 & \\
Positive & 142 & 20.7 \\
Negative & & 79.3 \\
\hline MUC1 & 102 & \\
Positive & 71 & 59.0 \\
Negative & & 41.0 \\
\hline Ki-67 & 113 & 62.1 \\
Low & 30 & 16.5 \\
Moderate & 39 & 21.4 \\
High & & \\
\hline Cyclin D & 81 & 44.8 \\
Positive & 100 & 55.2 \\
Negative & & \\
\hline Molecular subtype & 32 & 9.9 \\
Luminal A & 16 & 54.4 \\
Luminal B & 26 & \\
HER2 overexpression & 88 & \\
Triple negative & & \\
\hline
\end{tabular}

cytoplasmic MUC1 expression and cyclin D, and consequently, an association with poor prognostic factors.

Relating MUC1 expression pattern of our cases to the molecular subtypes revealed significant associations with two poor prognostic phenotypes. MUC1 overexpression was significantly exhibited by cases which were of the HER2 overexpression phenotype $\left(\chi^{2}=7.057, p=0.01\right)$. Although HER2 overexpression tumours are generally sensitive to trastuzumab therapy, they are inherently aggressive, with high proliferative rates, high histological grades, and frequent lymph node metastasis $[43,44]$. None of the previous Western studies which were reviewed reported a significant association between MUC1 expression and HER2 overexpression [33, 34, 36]. Elseed et al. [40] made no report on the association between MUC1 and HER2 expression, as their study focused only on ER status and the triple negative phenotype. Our study is therefore the first known to us to report such an association in a cohort of African cases.

The triple-negative subtype also demonstrated a significant association with MUC1 overexpression $\left(\chi^{2}=4.345\right.$, $p<0.05)$. The triple-negative phenotype is the predominant type portrayed by African women [45-47] and is characterized by aggressive histopathology, with high histological grades, high rates of proliferation, lymph node involvement, and distant metastasis. Do et al. [36] found an inverse association, where MUC1 negativity was rather predominant 


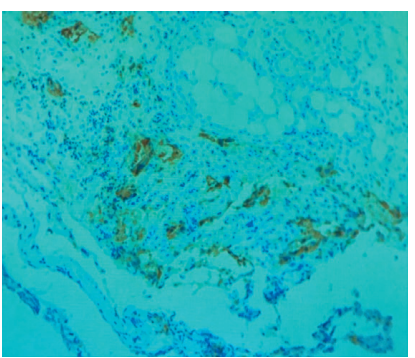

(a)

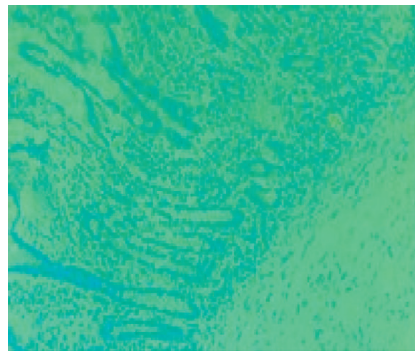

(b)

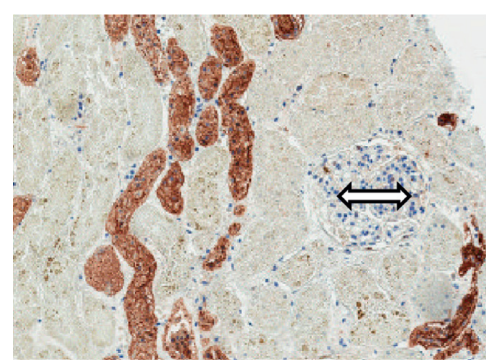

(c)

FIgUre 1: (a) Positive control (colonic tumour), (b) negative control (healthy colon tissue), and (c) negative internal control (breast cancer (nontumour, closed arrow)) for tumour-associated MUC1. The golden brown colours signify positive results.

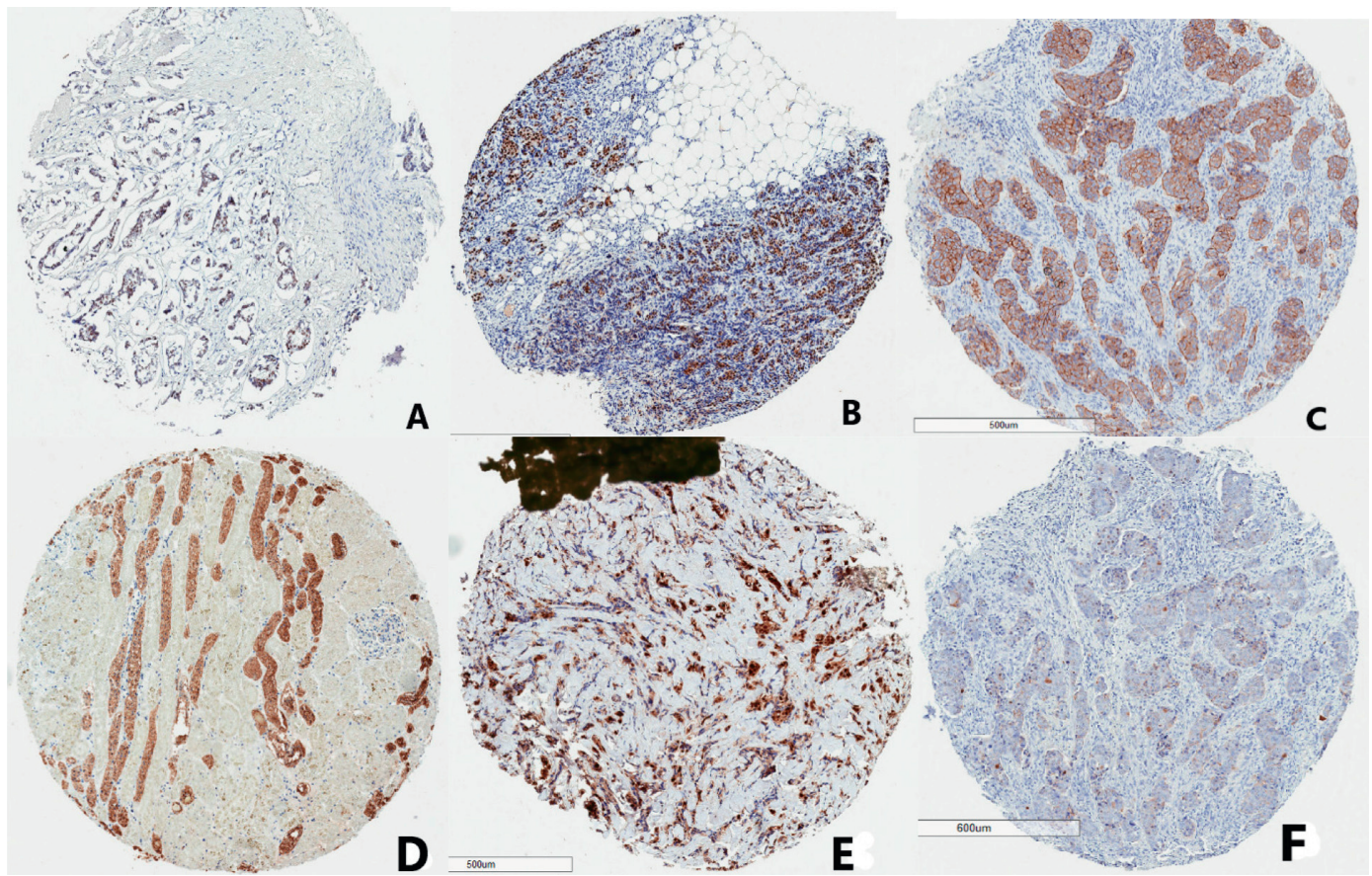

FIgURE 2: Photomicrographs of cases positive for ER (A), PR (B), HER2 (C), MUC1 (D), cyclin D (E), and high Ki-67 (F) immunohistochemistry. The golden brown colours signify positive tumour areas.

TABLE 4: Statistical analysis of MUC1 expression in relation to other parameters.

\begin{tabular}{|c|c|c|c|}
\hline & MUC1 positive (\%) & Chi-squared & $P$ value \\
\hline \multicolumn{4}{|c|}{ Clinicopathological characteristics } \\
\hline Age & & 38.412 & 0.810 \\
\hline Histological diagnosis & & 8.993 & 0.533 \\
\hline \multicolumn{4}{|l|}{ Histological grade } \\
\hline I & 53.8 & & \\
\hline II & 61.4 & 0.236 & 0.889 \\
\hline III & 59.4 & & \\
\hline Lymphovascular invasion & 63.0 & 0.639 & 0.575 \\
\hline \multicolumn{4}{|l|}{ Molecular subtypes } \\
\hline Luminal A & 60.7 & 0.122 & 0.828 \\
\hline Luminal B & 68.8 & 0.207 & 0.787 \\
\hline HER2 overexpression & 87.5 & 7.053 & 0.01 \\
\hline Triple negative & 56.6 & 4.345 & $<0.05$ \\
\hline \multicolumn{4}{|l|}{ Other markers } \\
\hline Cyclin D & 75.3 & 16.345 & $<0.001$ \\
\hline \multicolumn{4}{|l|}{$\mathrm{Ki}-67$} \\
\hline Low & 53.5 & & \\
\hline Moderate & 69.0 & 5.652 & 0.059 \\
\hline High & 73.7 & & \\
\hline
\end{tabular}


among the triple-negative cases in their cohort, while other studies found no such an association [39]. The sole prior study on MUC1 among Africans [40] found no association, although MUC1 was widely overexpressed and the cases were predominantly triple negative. Their inability to identify an association may however be explained by the small sample size used, which could not afford the study the desired statistical significance.

\section{Conclusion}

MUC1 was seen to be overexpressed in more than half of the cases tested. These tumours overexpressing MUC1 generally had high histological grades and high Ki-67 and cyclin D expression and were frequently involved in lymph node metastasis. Significant associations were seen between MUC1 and the HER2 overexpression, as well as the triplenegative subtypes.

This study therefore implies that MUC1 overexpression in breast cancer in Kumasi predicts poor prognosis, with aggressive tumour types that may be highly associated with metastasis.

\section{Data Availability}

The excel data used to support the findings of this study may be released upon application to the Committee on Human Research, Publication and Ethics of School of Medical Sciences/Komfo Anokye Teaching Hospital, Block J, School of Medical Sciences, Kwame Nkrumah University of Science and Technology, Kumasi, Ghana

\section{Conflicts of Interest}

The authors declare that they have no conflicts of interest.

\section{Acknowledgments}

The authors acknowledge the support offered by staff of the Department of Pathology, KATH, and the Department of Physiology. The assistance offered by Dr Vincent Dedu in the statistical analysis is also acknowledged. The authors also acknowledge Dr Frank Schneider and Ms Diane Lawson of Emory Hospital, Atlanta, Georgia, USA, for their assistance with IHC.

\section{References}

[1] F. Bray, J. Ferlay, I. Soerjomataram, R. L. Siegel, L. A. Torre, and A. Jemal, "Global cancer statistics 2018: GLOBOCAN estimates of incidence and mortality worldwide for 36 cancers in 185 countries," CA: A Cancer Journal for Clinicians, vol. 68, no. 6 , pp. 394-424, 2018.

[2] V. Vanderpuye, S. Grover, N. Hammad et al., "An update on the management of breast cancer in Africa," Infectious Agents and Cancer, vol. 12, no. 1, p. 13, 2017.

[3] K. D. Miller, R. L. Siegel, C. C. Lin et al., "Cancer treatment and survivorship statistics, 2016," CA: A Cancer Journal for Clinicians, vol. 66, no. 4, pp. 271-289, 2016.

[4] M. Sant, M. D. C. Lopez, R. Agresti et al., "Survival of women with cancers of breast and genital organs in Europe 1999-
2007: results of the EUROCARE-5 study," European Journal of Cancer, vol. 51, no. 15, pp. 2191-2205, 2015.

[5] B. M. Duduyemi, T. A. Adegbola, O. Ipadeola et al., "Expression of oestrogen receptors $\alpha$ and $\beta$ in primary and recurrent breast cancers," International Research Journal of Medicine and Medical Sciences, vol. 5, no. 2, pp. 25-36, 2014.

[6] C. Desantis, J. Ma, L. Bryan, and A. Jemal, "Breast cancer statistics, 2013," CA: A Cancer Journal for Clinicians, vol. 64, no. 1, pp. 52-62, 2013.

[7] B. Huang, M. Warner, and J.-Å. Gustafsson, "Estrogen receptors in breast carcinogenesis and endocrine therapy," Molecular and Cellular Endocrinology, vol. 418, pp. 240-244, 2015.

[8] F. Lumachi, G. Luisetto, M. M. S. Basso, U. Basso, A. Brunello, and V. Camozzi, "Endocrine therapy of breast cancer," Current Medicinal Chemistry, vol. 18, no. 4, pp. 513-522, 2011.

[9] G. Von Minckwitz, M. Procter, E. de Azambuja et al., "Adjuvant pertuzumab and trastuzumab in early her2-positive breast cancer," New England Journal of Medicine, vol. 377, no. 2, pp. 122-131, 2017.

[10] S. Escrivá-de-Romaní, M. Arumí, M. Bellet, and C. Saura, "HER2-positive breast cancer: current and new therapeutic strategies," The Breast, vol. 39, pp. 80-88, 2018.

[11] M. A. Molina, J. Codony-Servat, J. Albanell, F. Rojo, J. Arribas, and J. Baselga, "Trastuzumab (Herceptin), a humanized anti-HER2 receptor monoclonal antibody, inhibits basal and activated HER2 ectodomain cleavage in breast cancer cells," Cancer Research, vol. 61, no. 12, pp. 4744-4749, 2001.

[12] R. L. Siegel, K. D. Miller, and A. Jemal, "Cancer statistics, 2016,” CA: A Cancer Journal for Clinicians, vol. 66, no. 1, pp. 7-30, 2016.

[13] A. Siroy, F. W. Abdul-Karim, J. Miedler et al., "MUC1 is expressed at high frequency in early-stage basal-like triplenegative breast cancer," Human Pathology, vol. 44, no. 10, pp. 2159-2166, 2013.

[14] L. A. Brinton, J. D. Figueroa, B. Awuah et al., "Breast cancer in sub-Saharan Africa: opportunities for prevention," Breast Cancer Research and Treatment, vol. 144, no. 3, pp. 467-478, 2014.

[15] L. Brinton, fnm Lingua::EN::Titlecase, J. Figueroa et al., "Factors contributing to delays in diagnosis of breast cancers in Ghana, West Africa," Breast Cancer Research and Treatment, vol. 162, no. 1, pp. 105-114, 2017.

[16] J. J. Pitt, M. Riester, Y. Zheng et al., "Characterization of Nigerian breast cancer reveals prevalent homologous recombination deficiency and aggressive molecular features," Nature Communications, vol. 9, no. 1, p. 4181, 2018.

[17] L. A. Newman, "Disparities in breast cancer and African ancestry: a global perspective," The Breast Journal, vol. 21, no. 2, pp. 133-139, 2015.

[18] C. Denkert, C. Liedtke, A. Tutt, and G. von Minckwitz, "Molecular alterations in triple-negative breast cancer-the road to new treatment strategies," The Lancet, vol. 389, no. 10087, pp. 2430-2442, 2017.

[19] G. Bianchini, J. M. Balko, I. A. Mayer, M. E. Sanders, and L. Gianni, "Triple-negative breast cancer: challenges and opportunities of a heterogeneous disease," Nature Reviews Clinical Oncology, vol. 13, no. 11, pp. 674-690, 2016.

[20] C. Allemani, H. K. Weir, H. Carreira et al., "Global surveillance of cancer survival 1995-2009: analysis of individual data for 25676887 patients from 279 population-based registries in 67 countries (CONCORD-2)," The Lancet, vol. 385, no. 9972, pp. 977-1010, 2015. 
[21] A. C. Mensah, J. Yarney, S. K. Nokoe, S. Opoku, and J. N. Clegg-Lamptey, "Survival outcomes of breast cancer in Ghana: an analysis of clinicopathological features," OALib, vol. 03, no. 01, pp. 1-11, 2016.

[22] S. Nath and P. Mukherjee, "MUC1: a multifaceted oncoprotein with a key role in cancer progression," Trends in Molecular Medicine, vol. 20, no. 6, pp. 332-342, 2014.

[23] R. Singh and D. Bandyopadhyay, "MUC1: a target molecule for cancer therapy," Cancer Biology \& Therapy, vol. 6, no. 4, pp. 481-486, 2007.

[24] M. A. Cheever, J. P. Allison, A. S. Ferris et al., "The prioritization of cancer antigens: a National Cancer Institute pilot project for the acceleration of translational research," Clinical Cancer Research, vol. 15, no. 17, pp. 5323-5337, 2009.

[25] A. M. Sousa, P. M. Grandgenett, L. David, R. Almeida, M. A. Hollingsworth, and F. Santos-Silva, "Reflections on MUC1 glycoprotein: the hidden potential of isoforms in carcinogenesis," Apmis, vol. 124, no. 11, pp. 913-924, 2016.

[26] V. Apostolopoulos, L. Stojanovska, and S. E. Gargosky, "MUC1 (CD227): a multi-tasked molecule," Cellular and Molecular Life Sciences, vol. 72, no. 23, pp. 4475-4500, 2015.

[27] M. S. Syrkina, Y. S. Vassetzky, and M. A. Rubtsov, "MUC1 story: great expectations, disappointments and the renaissance," Current Medicinal Chemistry, vol. 26, no. 3, pp. 554-563, 2019.

[28] O. Finn and L. Zhang, "MUC1," Cancer Therapeutic Targets, vol. 2, pp. 391-398, 2017.

[29] M. Hossain and K. Wall, "Immunological evaluation of recent MUC1 glycopeptide cancer vaccines," Vaccines, vol. 4, no. 3, p. 25, 2016.

[30] S. K. Lau, L. M. Weiss, and P. G. Chu, "Differential expression of MUC1, MUC2, and MUC5AC in carcinomas of various sites," American Journal of Clinical Pathology, vol. 122, no. 1, pp. 61-69, 2004.

[31] H. Ideo, Y. Hinoda, K. Sakai et al., "Expression of mucin 1 possessing a $3^{\prime}$-sulfated core 1 in recurrent and metastatic breast cancer," International Journal of Cancer, vol. 137, no. 7, pp. 1652-1660, 2015.

[32] E. A. Rakha, R. W. G. Boyce, D. Abd El-Rehim et al., "Expression of mucins (MUC1, MUC2, MUC3, MUC4, MUC5AC and MUC6) and their prognostic significance in human breast cancer," Modern Pathology, vol. 18, no. 10, pp. 1295-1304, 2005.

[33] B. Van Der Vegt, M. A. J. de Roos, J. L. Peterse et al., "The expression pattern of MUC1 (EMA) is related to tumour characteristics and clinical outcome of invasive ductal breast carcinoma," Histopathology, vol. 51, no. 3, pp. 322-335, 2007.

[34] D. Kim, W.-H. Jung, and J. S. Koo, "Expression of MUC1, MUC2, MUC5AC and MUC5B in mucinous lesions of the breast," Pathobiology, vol. 79, no. 3, pp. 144-153, 2012.

[35] B. M. Syed, A. R. Green, E. C. Paish et al., "Biology of primary breast cancer in older women treated by surgery: with correlation with long-term clinical outcome and comparison with their younger counterparts," British Journal of Cancer, vol. 108, no. 5, pp. 1042-1051, 2013.

[36] S.-I. Do, K. Kim, D.-H. Kim et al., "Associations between the expression of mucins (MUC1, MUC2, MUC5AC, and MUC6) and clinicopathologic parameters of human breast ductal carcinomas," Journal of Breast Cancer, vol. 16, no. 2, pp. 152-158, 2013.

[37] WHO, WHO Classification of Tumours of the Breast, Fourth, International Agency for Research on Cancer (IARC), Lyon, France, 2012.
[38] N. A. Titiloye, A. Foster, G. O. Omoniyi-Esan et al., "Histological features and tissue microarray taxonomy of Nigerian breast cancer reveal predominance of the high-grade triplenegative phenotype," Pathobiology, vol. 83, no. 1, pp. 24-32, 2016.

[39] D. S. Patel, S. G. S. Khandeparkar, A. R. Joshi et al., "Immunohistochemical study of MUC1, MUC2 AND MUC5AC expression in primary breast Carcinoma," Journal of Clinical and Diagnostic Research, vol. 11, no. 4, pp. 30-34, 2017.

[40] S. M. H. Elseed, M. Siddig Abdelaziz, N. S. Salih, M. M. Elnaeem, M. El-Fatih, and A. I. Mudawi, "Mucin 1(MUC1) over expression and intracellular localization pattern in invasive ductal breast cancer using immunohistochemistry," American journal of research communication, vol. 3, no. 7, 2015.

[41] R. Love, "Cyclin D1 and P27KIP1: the gatekeepers of dysplasia," Journal of Immunological Sciences, vol. 2, no. 3, pp. 30-39, 2018.

[42] X. Liu, T. C. Caffrey, M. M. Steele et al., "MUC1 regulates cyclin D1 gene expression through p120 catenin and $\beta$-catenin," Oncogenesis, vol. 3, no. 6, p. e107, 2014.

[43] J. Makki, "Diversity of breast carcinoma: histological subtypes and clinical relevance," Clinical Medicine Insights: Pathology, vol. 8, no. 8, pp. 23-31, 2015.

[44] X. Dai, T. Li, Z. Bai et al., "Breast cancer intrinsic subtype classification, clinical use and future trends," American Journal of Cancer Research, vol. 5, no. 10, pp. 2929-2943, 2015.

[45] B. Seshie, N. A. Adu-Aryee, F. Dedey, B. Calys-Tagoe, and J.-N. Clegg-Lamptey, "A retrospective analysis of breast cancer subtype based on ER/PR and HER2 status in Ghanaian patients at the Korle Bu Teaching Hospital, Ghana," BMC Clinical Pathology, vol. 15, no. 1, p. 14, 2015.

[46] E. M. Der, R. K. Gyasi, Y. Tettey et al., "Triple-negative breast cancer in Ghanaian women: the Korle Bu Teaching Hospital experience," The Breast Journal, vol. 21, no. 6, pp. 627-633, 2015.

[47] K. G. Minoza, K. T. Yawe, Z. Mustapha, H. U. Na'aya, and H. A. Nggada, "“Hormonal and HER2 receptor immunohistochemistry of breast cancer in north-eastern Nigeria : a preliminary report," IOSR Journal of Dental and Medical Sciences, vol. 15, no. 6, pp. 18-23, 2016. 